\title{
Low Bone Turnover Markers in Young and Middle-Aged Male Patients with Type 2 Diabetes Mellitus
}

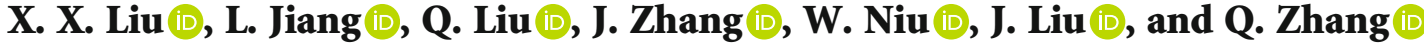 \\ NHC Key Laboratory of Hormones and Development, Tianjin Key Laboratory of Metabolic Diseases, Chu Hsien-I Memorial Hospital \\ \& Tianjin Institute of Endocrinology, Tianjin Medical University, Tianjin 300134, China \\ Correspondence should be addressed to Q. Zhang; zxyzhangqiumei@126.com
}

Received 27 April 2020; Revised 10 July 2020; Accepted 28 July 2020; Published 10 August 2020

Academic Editor: Viral Shah

Copyright ( 92020 X. X. Liu et al. This is an open access article distributed under the Creative Commons Attribution License, which permits unrestricted use, distribution, and reproduction in any medium, provided the original work is properly cited.

\begin{abstract}
Accumulating evidence has supported an increased risk of osteoporotic fracture in postmenopausal women and elderly men diagnosed with diabetes mellitus. However, it is not uncommon for young and middle-aged male patients diagnosed with type 2 diabetes mellitus (T2DM) to suffer from osteopenia or osteoporosis. Few studies focused on this population group are available. The aim of this study is to evaluate bone metabolic status and investigate the influence of T2DM on bone metabolism in 30-50year-old men. Anthropometric assessment and blood samples were obtained from 160 patients with T2DM and 69 nondiabetic volunteers. Serum parathyroid hormone $(\mathrm{PTH})$ and bone turnover markers (BTMs), including serum procollagen type I Nterminal peptide (PINP), osteocalcin (OC), and $\beta$-cross-linked C-telopeptide of type I collagen ( $\beta$-CTX), were analysed. No significant differences were observed based on age, body mass index, systolic blood pressure, serum calcium, phosphorus, creatinine, total protein, and albumin levels when comparing T2DM and control groups. Fasting blood glucose, HbA1c, triglyceride (TG), total cholesterol, and low-density lipoprotein cholesterol were significantly increased, while high-density lipoprotein cholesterol was significantly decreased in the T2DM group. Compared with controls, diabetic patients showed lower serum PINP, OC, and PTH levels, whereas serum $\beta$-CTX levels were similar between the two groups. Moreover, HbA1c levels were positively correlated with PINP and inversely associated with PTH levels. TG levels were negatively correlated with OC or $\beta$-CTX levels. Furthermore, multiple linear regression revealed a positive correlation between HbAlc and PINP levels. These results also revealed a negative association between $\mathrm{HbAlc}$ and $\mathrm{PTH}$, and between TG and OC levels, even after adjusting for expected confounder factors. Collectively, these findings indicated that young and middle-aged male patients with T2DM showed a lower turnover state resulting from bone formation inhibition. Glucose and lipid metabolic disorders may affect bone formation through different pathways.
\end{abstract}

\section{Introduction}

Type 2 diabetes mellitus (T2DM) is a common, chronic metabolic disease caused by insufficient insulin secretion and/or activity, leading to chronic hyperglycaemia. Its high prevalence has resulted in a heavy burden on social, financial, and health care systems [1]. There is a large amount of evidence revealing an increased risk of fracture in diabetic patients, particularly hip fracture [2, 3]. Recent metaanalyses indicated that hip fracture risk increases 1.3-1.7 times in patients with T2DM $[4,5]$. In addition, studies had demonstrated that severe vertebral fracture in patients with T2DM was associated with increased all-cause mortality $[6$,
7]. Osteoporotic fracture has been increasingly recognized as another complication of T2DM. High morbidity and mortality make the two diseases be more serious global health problem. The association between osteoporosis and T2DM should be paid close attention.

Osteoporosis is a skeletal chronic metabolic disease characterized by low bone mass and destroyed bone microarchitecture, resulting in the high risk of fragility fracture [8]. Therefore, bone metabolism should be further studied in patients with T2DM. Bone metabolism is a dynamic cyclical process where osteoblasts are involved in bone formation, and osteoclasts are involved in bone resorption [9]. Metabolites known as bone turnovers markers (BTMs) are generated 
from bone tissue and cells during the dynamic process and reflect bone metabolism during a relatively short period of time [9] and are thus better at predicting more recent changes. Specifically, procollagen type I N-terminal peptide (PINP) is the degradation product during the formation of type I collagen secreted by osteoblasts; serum osteocalcin (OC) is released by osteoblasts during bone formation; $\beta$ cross-linked C-telopeptide of type I collagen $(\beta$-CTX) is a breakdown product during the degradation of mature type I collagen secreted by osteoclasts [9]. Consequently, PINP and OC are key markers of bone formation and $\beta$-CTX is a key marker for bone resorption. The International Osteoporosis Foundation (IOF) recommends PINP and $\beta$-CTX as the reference markers for bone formation and bone resorption, respectively, due to their high sensitivity and specificity [10]. Recently, these BTMs have been used to assess bone metabolism, evaluate the clinical efficacy of osteoporosis therapies, and predict fracture risk [11]. Additionally, BTMs are shown to be associated with energy metabolism [12], which is closely related to glucose metabolism. Studying the effect of glucose metabolism disorders on BTMs is important to evaluate bone metabolic status in T2DM.

Most research has focused on studying postmenopausal women and elderly men since these two groups of individuals are at a high risk for fractures, especially those diagnosed with T2DM. Bone formation and bone mass are highest in the third decade and then decrease with age $[13,14]$. However, osteopenia or osteoporosis in young and middle-aged male patients with T2DM is not uncommon in clinical practice. Yet, only a few studies focused on these population groups are available. It is important to study how bone metabolism disorders affect younger patients with T2DM. Therefore, young and middle-aged male patients with T2DM were recruited as the subjects in the study presented here. We aim to assess bone metabolism by determining serum PINP, OC, $\beta$-CTX, and parathyroid hormone (PTH) levels and investigate the association among these markers and glucose metabolism. The goal is to explore the influence of T2DM on bone metabolism, which may allow for an accurate assessment of fracture risk and an earlier management of bone metabolism disorders.

\section{Materials and Methods}

2.1. Participants. The study presented here is a crosssectional study conducted in men aged 30-50 years old. Patients with T2DM, who were admitted to the Tianjin Metabolic Diseases Hospital from December 2017 to February 2018, were included in the T2DM group. Nondiabetic, male volunteers from the physical examination centre were recruited and included in the control group during the same period.

The diagnosis of T2DM was based on the guidelines provided by the World Health Organization [15] including: fasting blood glucose (FBG) level $\geq 7.0 \mathrm{mmol} / \mathrm{l}$ $(126 \mathrm{mg} / \mathrm{dl})$ or $2 \mathrm{~h}$ blood glucose $\geq 11.1 \mathrm{mmol} / \mathrm{l}(199 \mathrm{mg} / \mathrm{dl})$ during an oral glucose tolerance test (OGTT). Diabetic patients were treated with oral antidiabetic agents or in combination with insulin. Exclusion criteria included the presence of kidney disease $\left(e G F R<60 \mathrm{~mL} / \mathrm{min} / 1.73 \mathrm{~m}^{2}\right)$, hepatic disease (ALT or AST $\geq 2.5$ times than the upper reference), cancer, rheumatic diseases (rheumatic arthritis and rheumatoid arthritis), other bone metabolic diseases (osteitis and osteomalacia), hypercalcemia, or other endocrine diseases (Cushing's syndrome, primary hyperparathyroidism, and thyroid dysfunction). Participants taking medications that may influence bone metabolism were also excluded. These medications included glucocorticoids, calcium, vitamin D, antiosteoporosis drugs, steroids, and thyroid hormones.

This study was conducted following the Declaration of Helsinki (1964) and was approved by the Ethics Committee of the Tianjin Medical University Chu Hsien-I Memorial Hospital. Each participant signed a written informed consent form.

2.2. Clinical Measurements. Anthropometric and biochemical assessments were performed in all participants. Diabetic duration, height, weight, body mass index (BMI), and blood pressure data were collected. BMI was calculated by the formula as weight (in $\mathrm{kg}$ ) divided by height squared (in $\mathrm{m}^{2}$ ). All overnight fasting blood samples were obtained in the morning. Serum samples were separated by centrifugation and stored at $-80^{\circ} \mathrm{C}$. Blood calcium, phosphorus, total protein, albumin, alanine aminotransferase, aspartate aminotransferase, alkaline phosphatase (ALP), serum creatinine, uric acid, urea nitrogen, haemoglobin A1c (HbA1c), FBG, insulin, C-peptide, total cholesterol (TC), triglyceride (TG), high-density lipoprotein cholesterol (HDL-c), and low-density lipoprotein cholesterol (LDL-c) were measured using standard methods. Serum parathyroid hormone (PTH) and BTM levels, including PINP, OC, and $\beta$-CTX, were measured using an IDS-iSYS automated analyser (Roche, Germany). The intra-assay and interassay coefficients of variation (CVs) of BTMs were below $5 \%$ and $7 \%$, respectively.

2.3. Statistical Analyses. The statistical analyses were performed with SPSS 20.0 (SPSS Inc., Chicago, IL, USA). Normality testing was conducted in all continuous variables. Variables with normal distributions were described as mean \pm standard deviation, and the differences were determined using Student's $t$-test between the two groups. Those with skewed distributions were expressed as median (interquartile range), and differences between groups were assessed using the Mann-Whitney $U$ test. The Pearson or Spearman correlation analysis was used to determine the correlation between blood glucose or lipid and bone metabolism markers. Multiple linear regression analyses were conducted to evaluate the association between HbA1c, TG, and BTMs. $P$ value $<0.05$ was considered statistically significant.

\section{Results}

A total of 160 diabetic patients were included in the T2DM group. The mean age of these patients was $41.0 \pm 5.9$ years and the mean diabetic duration was 4.0 years (ranging from 1.0 to 9.0 years). The mean FBG was $8.64 \pm 2.40 \mathrm{mmol} / \mathrm{l}$, 
TABle 1: Comparison of characteristics between diabetic patients and controls.

\begin{tabular}{|c|c|c|c|}
\hline Variables & $\begin{array}{l}\text { Patients with T2DM } \\
\qquad(n=160)\end{array}$ & $\begin{array}{c}\text { Nondiabetic } \\
\text { controls }(n=69)\end{array}$ & $P$ \\
\hline Age $(y)$ & $41.0 \pm 5.9$ & $41.8 \pm 5.1$ & 0.285 \\
\hline $\begin{array}{l}\text { Diabetic } \\
\text { duration }(y)\end{array}$ & $4.0(1.0,9.0)$ & - & - \\
\hline Height $(\mathrm{cm})$ & $173.7 \pm 6.7$ & $172.8 \pm 4.5$ & 0.220 \\
\hline Weight (kg) & $84.4 \pm 15.0$ & $81.4 \pm 11.1$ & 0.097 \\
\hline BMI $\left(\mathrm{kg} / \mathrm{m}^{2}\right)$ & $27.89 \pm 4.28$ & $27.27 \pm 3.68$ & 0.294 \\
\hline SBP (mmHg) & $129.6 \pm 13.0$ & $127.8 \pm 13.0$ & 0.347 \\
\hline DBP (mmHg) & $80.8 \pm 9.6$ & $79.8 \pm 9.0$ & 0.470 \\
\hline FBG (mmol/l) & $8.64 \pm 2.40$ & $5.09 \pm 0.27$ & $<0.001$ \\
\hline HbAlc (\%) & $8.69 \pm 1.84$ & - & - \\
\hline INS (mIU/l) & $9.28(5.32,13.73)$ & - & - \\
\hline C-P (ng/ml) & $1.59(1.15,2.16)$ & - & - \\
\hline $\mathrm{TG}(\mathrm{mmol} / \mathrm{l})$ & $2.32(1.42,3.23)$ & $1.22(0.85,1.86)$ & $<0.001$ \\
\hline $\mathrm{TC}(\mathrm{mmol} / \mathrm{l})$ & $5.08 \pm 1.12$ & $4.00 \pm 0.94$ & $<0.001$ \\
\hline $\begin{array}{l}\text { HDL-c } \\
(\mathrm{mmol} / \mathrm{l})\end{array}$ & $1.06 \pm 0.24$ & $1.16 \pm 0.33$ & 0.020 \\
\hline $\begin{array}{l}\text { LDL-c } \\
(\mathrm{mmol} / \mathrm{l})\end{array}$ & $3.20 \pm 0.96$ & $2.43 \pm 0.78$ & $<0.001$ \\
\hline $\mathrm{Ca}(\mathrm{mmol} / \mathrm{l})$ & $2.31 \pm 0.13$ & $2.33 \pm 0.12$ & 0.193 \\
\hline $\mathrm{P}(\mathrm{mmol} / \mathrm{l})$ & $1.31 \pm 0.18$ & $1.26 \pm 0.18$ & 0.118 \\
\hline TP (g/l) & $68.70 \pm 4.22$ & $68.78 \pm 5.10$ & 0.897 \\
\hline $\operatorname{ALB}(g / l)$ & $43.98 \pm 3.17$ & $44.08 \pm 2.89$ & 0.825 \\
\hline ALT (IU/l) & $21.35 \pm 9.78$ & $20.97 \pm 9.04$ & 0.782 \\
\hline AST (IU/l) & $20.42 \pm 10.62$ & $19.59 \pm 7.15$ & 0.554 \\
\hline ALP (IU/l) & $70.9 \pm 18.37$ & $68.85 \pm 14.92$ & 0.410 \\
\hline $\mathrm{Cr}(\mu \mathrm{mol} / \mathrm{l})$ & $71.04 \pm 12.90$ & $71.71 \pm 8.25$ & 0.639 \\
\hline SUA $(\mu \mathrm{mol} / \mathrm{l})$ & $344.45 \pm 90.21$ & $339.44 \pm 60.07$ & 0.932 \\
\hline $\begin{array}{l}\text { BUN } \\
(\mathrm{mmol} / \mathrm{l})\end{array}$ & $5.26 \pm 1.17$ & $5.19 \pm 0.87$ & 0.641 \\
\hline
\end{tabular}

y: years; T2DM: type 2 diabetes mellitus; BMI: body mass index; SBP: systolic blood pressure; DBP: diastolic blood pressure; FBG: fasting blood glucose; HbAlc: haemoglobin A1c; INS: fasting insulin; C-P: fasting C-peptide; TG: triglyceride; TC: total cholesterol; HDL-c: high-density lipoprotein cholesterol; LDL-c: low-density lipoprotein cholesterol; Ca: calcium; P: phosphorus; TP: total protein; ALB: albumin; ALT: alanine aminotransferase; AST: aspartate aminotransferase; ALP: alkaline phosphatase; Cr: serum creatinine; SUA: serum uric acid; BUN: blood urea nitrogen. $P$ value $<0.05$ was considered significant.

and the mean $\mathrm{HbAlc}$ value was $8.69 \pm 1.84 \%$. A total of 69 nondiabetic volunteers were recruited in the control group that had a mean age of $41.8 \pm 5.1$ years and a mean FBG of $5.09 \pm 0.27 \mathrm{mmol} / \mathrm{l}$.

Baseline clinical characteristics of the two groups are shown in Table 1. No significant differences were observed between the control or T2DM groups for age $(P=0.285)$, height $(P=0.220)$, weight $(P=0.097)$, BMI $(P=0.294)$, or systolic blood pressure $(P=0.347)$. There were also no significant differences between the two groups for serum calcium
$(P=0.193)$, phosphorus $(P=0.118)$, creatinine $(P=0.639)$, total protein $(P=0.897)$, albumin $(P=0.825)$, or ALP $(P=0.410)$. As expected, patients in the T2DM group showed significantly higher FBG levels $(P<0.001)$ compared with the control group. In addition, significantly higher TG $(P<0.001)$, TC $(P<0.001)$, and LDL-c $(P<0.001)$ levels and significantly lower HDL-c $(P=0.020)$ levels were observed in diabetic patients compared with controls.

Comparison of BTMs and PTH between diabetic patients and controls is shown in Table 2. There were significant decreases in serum PINP $(P=0.008)$, OC $(P<0.001)$, and PTH $(P=0.001)$ levels in patients with T2DM compared with controls. In contrast, serum $\beta$-CTX levels were similar between the two groups $(P=0.826)$.

Moreover, univariate correlation analyses were performed to investigate the association between blood glucose or lipid and bone metabolism markers. The results revealed that $\mathrm{HbAlc}$ was positively correlated with PINP ( $\mathrm{rs}=0.171$, $P=0.030)$ and inversely associated with PTH $(r=-0.176$, $P=0.026$ ). There was a significant negative correlation between OC or $\beta$-CTX and TG (rs $=-0.164, P=0.038$; rs $=$ $-0.173, P=0.028$ ) levels (Figure 1). There was no significant association observed between PINP and TG, or between OC and $\mathrm{HbAlc}$ levels. Age was negatively correlated with PINP (rs $=-0.269, P=0.001)$, OC ( $\mathrm{rs}=-0.168, P=0.033$ ), and PTH ( $r=0.164, P=0.038)$, but not with $\beta$-CTX levels. The BTMs and PTH levels did not correlate with BMI, blood pressure, calcium, or phosphorous levels.

Furthermore, multiple linear regression analyses were performed to examine the cross-sectional association between blood glucose or lipid and BTMs after adjusting for expected confounder factors. Serum PINP, OC, or PTH levels were used as dependent variables, while HbAlc or TG levels were used as independent variables. These findings indicated that $\mathrm{HbAlc}$ was positively correlated with PINP $(\beta=0.229, P=0.004)$ and inversely associated with PTH $(\beta=-0.213, P=0.011)$ after adjusting for age, BMI, systolic blood pressure, TG, HDL-c, LDL-c, calcium, and phosphorus. Our results also showed a significant negative correlation between TG and OC $(\beta=-0.177, P$ $=0.034)$ after adjusting for age, BMI, systolic blood pressure, HbA1c, HDL-c, LDL-c, calcium, and phosphorus (Table 3). All independent variables used in multiple linear analyses are shown in Table S1.

\section{Discussion}

Most previous studies investigating postmenopausal women and elderly men have shown that the markers for bone formation and/or resorption were reduced in patients with T2DM, compared with nondiabetic individuals [16], indicating a lower bone turnover state. It is unclear whether young and middle-aged diabetic patients shared similar results. In this study, we focused on young and middleaged male patients with T2DM. Results demonstrated that diabetic patients had significantly lower serum PINP and OC levels compared with the control individuals. In contrast, serum $\beta$-CTX levels were not significantly different between the two groups. Results indicated that inhibition 
TABLE 2: Comparison of BTMs and PTH between diabetic patients and controls.

\begin{tabular}{lccc}
\hline Variables & Patients with T2DM & Nondiabetic controls & $P$ \\
\hline PINP $(\mathrm{ng} / \mathrm{ml})$ & $33.38(22.53,46.02)$ & $41.33(30.40,48.51)$ & 0.008 \\
OC $(\mathrm{ng} / \mathrm{ml})$ & $5.74(4.92,7.55)$ & $7.14(5.64,9.18)$ & $<0.001$ \\
$\beta$-CTX $(\mathrm{ng} / \mathrm{ml})$ & $0.19(0.12,0.30)$ & $0.20(0.14,0.27)$ & 0.826 \\
PTH $(\mathrm{pg} / \mathrm{ml})$ & $25.21 \pm 9.13$ & $29.86 \pm 11.13$ & 0.001 \\
\hline
\end{tabular}

T2DM: type 2 diabetes mellitus; PINP: procollagen type I N-terminal peptide; OC: osteocalcin; $\beta$-CTX: $\beta$-cross-linked C-telopeptide of type I collagen; PTH: parathyroid hormone. $P$ value $<0.05$ was considered significant.

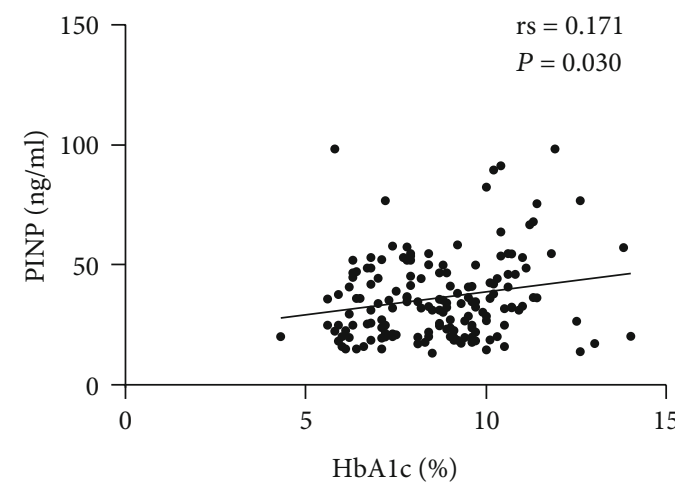

(a)

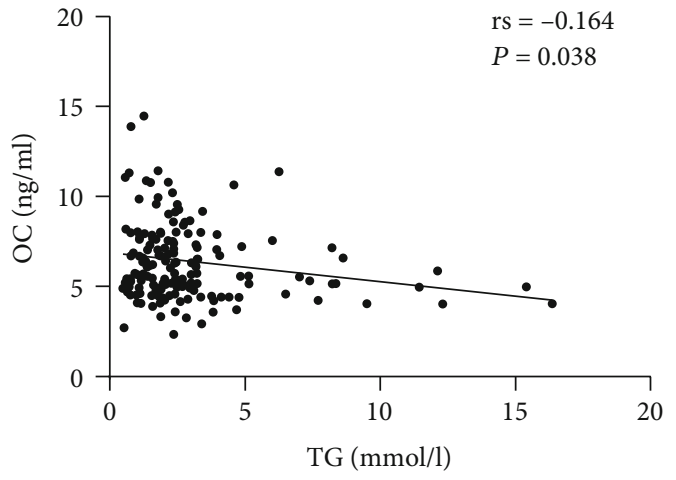

(c)

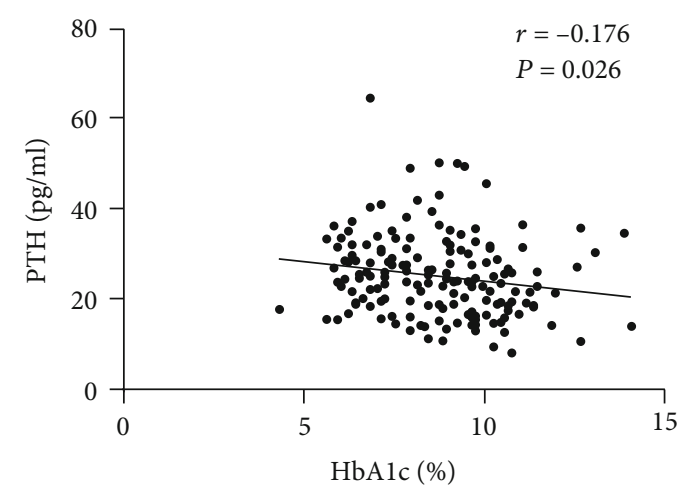

(b)

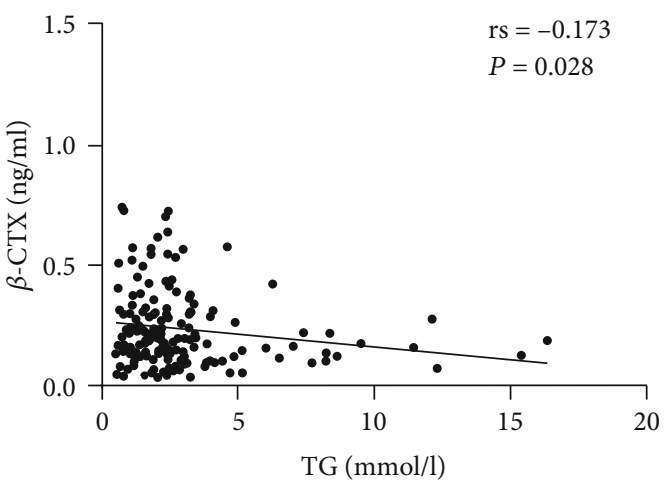

(d)

Figure 1: Correlation between serum glucose or lipid levels and BTMs or PTH. (a) Correlation between PINP and HbA1c. (b) Correlation between PTH and HbA1c. (c) Correlation between OC and TG. (d) Correlation between $\beta$-CTX and TG. HbA1c: haemoglobin A1c; TG: triglyceride; PINP: procollagen type I N-terminal peptide; OC: osteocalcin; $\beta$-CTX: $\beta$-cross-linked C-telopeptide of type I collagen; PTH: parathyroid hormone. $r$ : Pearson's correlation coefficient; rs: Spearman's correlation coefficient. $P$ value $<0.05$ was considered significant.

TABLE 3: Multiple linear regression analyses between serum glucose or lipid and bone metabolism markers.

\begin{tabular}{|c|c|c|c|c|}
\hline Dependent variable & Independent variables & Unstandardized coefficients $(\beta)$ & Standardized coefficients $(\beta)$ & $P$ \\
\hline PINP & $\mathrm{HbA1c}$ & 2.140 & 0.229 & 0.004 \\
\hline OC & TG & -0.144 & -0.177 & 0.034 \\
\hline PTH & $\mathrm{HbAlc}$ & -1.054 & -0.213 & 0.011 \\
\hline
\end{tabular}

HbAlc: haemoglobin A1c; TG: triglyceride; PINP: procollagen type I N-terminal peptide; OC: osteocalcin; PTH: parathyroid hormone. $P$ value $<0.05$ was considered significant.

of bone formation phase, rather than resorption, led to a lower bone turnover state in young and middle-aged male patients with T2DM. Moreover, this study demonstrated that $\mathrm{HbAlc}$ was an independent factor for PINP, suggest- ing the influence of glycaemic control on PINP in young and middle-aged male patients with T2DM. Early glycaemic control may reduce the risk of fracture by delaying bone formation reduction. 
Reduced serum OC levels were previously reported in male patients with T2DM [17-19]. Bezerra dos Santos Magalhaes et al. further demonstrated a weak negative correlation between FBG and OC levels [19]. Whereas serum PINP was not available in these studies. A recent study revealed a decrease in serum PINP levels in patients with impaired fasting glucose and diabetes [20], which was in line with our research. Further analyses revealed that serum PINP levels were significantly reduced in younger diabetic patients ( $<65$ years old) compared with the older patients ( $\geq 65$ years old), but serum $\beta$-CTX was also significantly decreased [20]. The controversial conclusions may be related to differences in age, race, diabetic duration, and glycaemic control. A study by Kulkarni et al. [18] shared a similar relationship between $\mathrm{HbAlc}$ and PINP levels. Additionally, a largescale cross-sectional study performed in Germany indirectly supported this conjecture. The authors revealed that chances for metabolic syndrome or T2DM significantly decreased with the higher serum PINP and $\beta$-CTX levels in men aged 25-55 years old [21]. However, two largescale studies performed in China, one involving men and women aged 30-60 years old [22] and the other including men aged 20-69 years old [23], indicated that serum OC was negatively correlated with chances for T2DM, even after adjusting age, BMI, waist circumference, blood pressure, FBG, and TG. As described by these studies, the close relationship between glucose and BTMs has been investigated but needs further understanding.

In addition, compared with controls, diabetic patients showed higher TG, TC, and LDL-c and lower HDL-c levels, which may represent a high probability of lipid metabolism disorders in patients with T2DM. Further analyses investigating the correlation between lipid and BTMs revealed a significant, negative correlation between serum TG and OC levels. High TG levels may reduce serum OC levels and inhibit bone formation in young and middle-aged male patients with T2DM. These observations were similar to what was found in a recent male population-based study where serum TG levels were also inversely correlated with OC levels [23]. Some research investigating male diabetic patients showed no relationship between serum OC levels and blood lipid metabolism $[24,25]$. These findings are contradictory to one another. Differences in age, race, and metabolic status may account for these controversial results.

The impact of blood glucose and lipid metabolism disorders on BTMs needs further studies to elucidate mechanisms. It is known that hyperglycaemia can lead to osmotic diuresis, which causes renal calcium leakage and a negative calcium balance. Improved blood glucose control contributes to the reduction of urinary calcium levels [26]. The calciumsensing defect and secondary chronic hypomagnesaemia induced by osmotic diuresis may be responsible for impaired PTH secretion [27]. The pathological regulation of PTH on BTMs in patients with T2DM is not clear. In this study, serum PTH levels were decreased and were negatively associated with HbAlc levels in T2DM, implying that diabetic patients, especially those with poor glycaemic control, had lower PTH levels. These observations were in line with previous studies $[28,29]$. Relative hypoparathyroidism may dis- rupt bone metabolism in patients with T2DM. Previous studies demonstrated that low PTH levels directly inhibited osteoblast activity and contribute to bone demineralization. In the nondiabetic population, PTH inhibited transcriptional suppression of sclerostin produced by osteocytes. As a Wnt antagonist, sclerostin inhibited $\mathrm{Wnt} / \beta$-catenin signalling and osteoblast activity. However, the regulation of PTH on sclerostin may be impaired in diabetes [30]. As mentioned above, the negative relationship between blood glucose and bone metabolism is probably explained.

Otherwise, chronic inflammatory conditions and turbulence of adipokines increased the risk of osteoporosis in patients with T2DM [31]. Advanced glycation end-products (AGEs) were accumulated in diabetes and played a key role in chronic inflammatory complications [32]. Previous studies have shown that BTMs were suppressed by hyperinsulinemia and the accumulation of AGEs [33]. AGEs promoted the production of both inflammatory cytokines and reactive oxygen species (ROS) in the body by activating ligands, further triggering chronic inflammation and bone resorption [34]. In vitro studies reported that AGE- 2 and AGE- 3 inhibited the maturation of human marrow mesenchymal stem cells (MSCs) and their differentiation into cartilage and bone tissues, resulting in decreased osteoblasts [35]. Moreover, the formation and accumulation of AGEs inhibited synthesis of osteocalcin and osteoblastic ossein matrix [36], increased nonenzymatic cross-linked folding of the collagen fibres [37], and disturbed osteoblast development. A recent study indicated that hyperglycaemia directly inhibited the differentiation of osteoblasts and then decreased bone formation, enhanced osteoclast activity, and increased bone absorption, eventually leading to a reduction of bone mass [38, 39]. Glucose and insulin signalling involved receptor activation of the nuclear factor $\kappa \mathrm{B}$ ligand/osteoprotegerin (RANKL/OPG) pathway $[40,41]$. Analyses revealed that lower serum RANKL levels were associated with higher TG levels [42]. This inverse relationship may explain the results generated in this study. Furthermore, adiponectin, a recently uncovered adipocytokine, is produced exclusivity in adipose tissue. Research shows that adiponectin stimulated osteoblast proliferation, differentiation, and mineralization [43]. However, serum adiponectin concentrations decreased in patients with T2DM [44]. The turbulence of adipocytokines may lead to an imbalance of bone metabolism.

Antidiabetic agents may have different effects on bone metabolism. Agents that may have an effect include thiazolidinediones (TZDs), sodium-glucose-linked transporter-2 (SGLT-2) inhibitors, insulin, and glucagon-like peptide-1 receptor agonists (GLP-1 RA). A previous work shows that rosiglitazone, a type of TZDs, promoted osteoblast/osteocyte apoptosis and led to a negative balance in bone metabolism [45]. Analyses demonstrated a gender difference when it came to the effects of TZDs on fracture in patients with T2DM and confirmed that TZDs only increased fracture risk in female patients and not male patients [46]. SGLT-2 inhibitors improved blood glucose levels by promoting urinary glucose excretion, which may affect urinary calcium excretion and bone metabolism. Canagliflozin treatment was associated with a higher fracture rate in patients with T2DM [47]. A 
meta-analysis indicated no relationship between three SGLT-2 inhibitors (canagliflozin, dapagliflozin, and empagliflozin) and fracture risk. Clinical studies on adverse skeletal events of SGLT-2 inhibitors are still lacking. Few studies have assessed the association between insulin injection and BTMs. Several studies reported an increased fracture risk in insulin-treated patients with T2DM [48]. A high incidence of hypoglycaemic events and falling [49] may be the main reasons in older adults. Long-term disease and the presence of multiple diabetic complications may also disrupt bone metabolism. No significant differences were observed between diabetic patients under treatment with $(n=35)$ or without $(n=125)$ TZDs, with $(n=10)$ or without $(n=150)$ SGLT-2 inhibitors, and with $(n=85)$ or without $(n=75)$ insulin in this study. Liraglutide and exenatide, two GLP-1 RAs, may improve skeletal blood supply, increase bone mineral density (BMD), and reduce the risk of osteoporosis and fracture in animal and human studies $[27,50]$. However, the bone protective effects behind this require clinical studies. There were no significant differences observed between diabetic patients under treatment with $(n=12)$ or without $(n=148)$ GLP-1 RAs in our study. In addition, there were also no significant differences between patients under treatment with $(n=54)$ or without $(n=106)$ dipeptidyl peptidase-4 (DPP-4) inhibitors, with $(n=42)$ or without $(n=118)$ insulin secretagogues, with $(n=108)$ or without $(n=52)$ metformin, and with $(n=94)$ or without $(n=66)$ alpha-glucosidase inhibitors (AGI) in the present study (Table S2). As a multiple metabolic disease, the treatment of T2DM is complex and requires additional clinical studies to evaluate the influence of these therapies on bone metabolism.

One advantage of this study is that it focused on male diabetic patients aged 30-50 years old, where BTMs varied with small changes and there was a restriction on gender and age being an influence on the results. With this, it was easier to investigate the relationship between blood glucose, lipids, and bone metabolism. However, this study still faces some limitations. First, the cross-sectional design prevents one from drawing a causal relationship and failed to explore changes in BTMs after improving blood glucose and lipid metabolism disorders. Further prospective research may offer additional information about this. Second, the sample size number between the two groups was unequal and the number of controls used was inadequate. Besides, this study was a single-centre study that only analysed patients with relatively severe diabetes. Therefore, the results presented here may not be generalizable to all young and middle-aged male populations diagnosed with T2DM. Large-scale and multicentre studies remained to verify these issues. Third, the influence of antidiabetic agents on bone metabolism remains contradictory. Consequently, potential confounder factors may exist. Fourth, serum levels of bone-specific alkaline phosphatase (BAP), vitamin D, or steroids, all of which influence bone metabolism, were not determined in this study. Serum ALP is mainly derived from liver isoform (LAP), and its specificity for bone metabolism is lacking [51]. BAP is a more bone-specific marker of bone formation, while the current immunoassays available for BAP still show up to $20 \%$ cross-reactivity toward LAP [52]. As recommended by the IOF [10], serum PINP was preferred for bone formation because of high specificity in our study. Vitamin D promotes the absorption of calcium and may affect bone metabolism. However, relatively limited data about the effect of vitamin $\mathrm{D}$ on BTMs are available. A prospective partial intervention study in postmenopausal women with T2DM shows that 25(OH)D was positively correlated with PINP, especially in patients taking alfacalcidol [53]. The MINOS study, a prospective study of 881 men aged 19-85 years, revealed that serum $25(\mathrm{OH}) \mathrm{D}$ was not associated with BTMs in men under 55 years of age $(n=286)$ [54]. The relationship between vitamin D and BTMs still needs further research. Fifth, we did not take BMD into consideration. BMD altogether with BTMs may be helpful to evaluate bone metabolism. BMD reflects mineral density of bone and is the cumulative result of long-term bone metabolic activities. This study mainly focused on the impact of T2DM on BTMs and evaluated the recent changes of bone metabolism. Further studies should be conducted to investigate the long-term effect of T2DM on BMD.

\section{Conclusions}

This study demonstrated that young and middle-aged male patients with T2DM showed a lower turnover state resulting from bone formation inhibition. HbA1c levels were positively correlated with PINP levels and inversely associated with PTH levels. These findings also revealed a negative correlation between TG and OC levels, even after adjusting for expected confounder factors. Glucose and lipid metabolism disorders may affect bone formation through different pathways. The study presented here provides evidence of T2DM influencing bone metabolism in young and middle-aged men. The improvement of blood glucose and lipids may be beneficial to bone metabolism and reduce fracture risk in patients with T2DM.

\section{Data Availability}

The data used to support the findings of this study are available from the corresponding author upon request.

\section{Conflicts of Interest}

The authors declare that there is no conflict of interest regarding the publication of this paper.

\section{Acknowledgments}

This work was supported by Scientific Research Funding of Tianjin Medical University Chu Hsien-I Memorial Hospital (grant numbers 2018ZDKF07). We gratefully acknowledge the participants in this study.

\section{Supplementary Materials}

Table S1: multiple linear regression between serum glucose or lipid levels and BTMs or PTH. Table S2: the information about the medications of the patients with T2DM. (Supplementary Materials) 


\section{References}

[1] K. Ogurtsova, J. D. da Rocha Fernandes, Y. Huang et al., "IDF Diabetes Atlas: Global estimates for the prevalence of diabetes for 2015 and 2040," Diabetes Research and Clinical Practice, vol. 128, pp. 40-50, 2017.

[2] A. V. Schwartz, D. E. Sellmeyer, K. E. Ensrud et al., "Older women with diabetes have an increased risk of fracture: a prospective study," Journal of Clinical Endocrinology and Metabolism, vol. 86, no. 1, pp. 32-38, 2001.

[3] L. Forsen, H. E. Meyer, K. Midthjell, and T. H. Edna, "Diabetes mellitus and the incidence of hip fracture: results from the Nord-Trøndelag Health Survey,” Diabetologia, vol. 42, no. 8, pp. 920-925, 1999.

[4] Y. Fan, F. Wei, Y. Lang, and Y. Liu, "Diabetes mellitus and risk of hip fractures: a meta-analysis," Osteoporosis International, vol. 27, no. 1, pp. 219-228, 2016.

[5] M. Janghorbani, R. M. Van Dam, W. C. Willett, and F. B. Hu, "Systematic review of type 1 and type 2 diabetes mellitus and risk of fracture," American Journal of Epidemiology, vol. 166, no. 5, pp. 495-505, 2007.

[6] I. Kostoglou-Athanassiou, P. Athanassiou, A. Gkountouvas, and P. Kaldrymides, "Vitamin D and glycemic control in diabetes mellitus type 2," Therapeutic Advances in Endocrinology and Metabolism, vol. 4, no. 4, pp. 122-128, 2013.

[7] H. Miyake, I. Kanazawa, and T. Sugimoto, "Association of bone mineral density, bone turnover markers, and vertebral fractures with all-cause mortality in type 2 diabetes mellitus," Calcified Tissue International, vol. 102, no. 1, pp. 1-13, 2018.

[8] E. S. Siris, R. Adler, J. Bilezikian et al., "The clinical diagnosis of osteoporosis: a position statement from the National Bone Health Alliance Working Group," Osteoporosis International, vol. 25, no. 5, pp. 1439-1443, 2014.

[9] M. B. Greenblatt, J. N. Tsai, and M. N. Wein, "Bone turnover markers in the diagnosis and monitoring of metabolic bone disease," Clinical Chemistry, vol. 63, no. 2, pp. 464-474, 2017.

[10] S. Vasikaran, for the IOF-IFCC Bone Marker Standards Working Group, R. Eastell et al., "Markers of bone turnover for the prediction of fracture risk and monitoring of osteoporosis treatment: a need for international reference standards," Osteoporosis International, vol. 22, no. 2, pp. 391-420, 2011.

[11] H. W. S. Cabral, B. F. G. Andolphi, B. V. C. Ferreira et al., "The use of biomarkers in clinical osteoporosis," Revista da Associação Médica Brasileira, vol. 62, no. 4, pp. 368-376, 2016.

[12] P. Iglesias, F. Arrieta, M. Piñera et al., "Serum concentrations of osteocalcin, procollagen type $1 \mathrm{~N}$-terminal propeptide and beta-CrossLaps in obese subjects with varying degrees of glucose tolerance," Clinical Endocrinology, vol. 75, no. 2, pp. 184-188, 2011.

[13] J. M. Wishart, A. O. Need, M. Horowitz, H. A. Morris $\dagger$, and B. E. C. Nordin, "Effect of age on bone density and bone turnover in men," Clinical Endocrinology, vol. 42, no. 2, pp. 141146, 1995.

[14] P. Szulc, P. Garnero, F. Munoz, F. Marchand, and P. D. Delmas, "Cross-sectional evaluation of bone metabolism in men," Journal of Bone and Mineral Research, vol. 16, no. 9, pp. 1642-1650, 2001.

[15] K. G. M. M. Alberti, P. Z. Zimmet, and WHO Consultation, "Definition, diagnosis and classification of diabetes mellitus and its complications. Part 1: diagnosis and classification of diabetes mellitus provisional report of a WHO consultation," Diabetic Medicine, vol. 15, no. 7, pp. 539-553, 1998.
[16] J. Starup-Linde and P. Vestergaard, "Biochemical bone turnover markers in diabetes mellitus - a systematic review," Bone, vol. 82, pp. 69-78, 2016.

[17] L. Achemlal, S. Tellal, F. Rkiouak et al., "Bone metabolism in male patients with type 2 diabetes," Clinical Rheumatology, vol. 24, no. 5, pp. 493-496, 2005.

[18] S. V. Kulkarni, S. Meenatchi, R. Reeta, R. Ramesh, A. R. Srinivasan, and C. Lenin, "Association of glycemic status with bone turnover markers in type 2 diabetes mellitus," International Journal of Applied \& Basic Medical Research, vol. 7, no. 4, pp. 247-251, 2017.

[19] K. B. dos Santos Magalhães, M. M. Magalhães, E. T. Diniz, C. S. Lucena, L. Griz, and F. Bandeira, "Metabolic syndrome and central fat distribution are related to lower serum osteocalcin concentrations," Annals of Nutrition and Metabolism, vol. 62, no. 3, pp. 183-188, 2013.

[20] K. L. Holloway-Kew, L. L. F. De Abreu, M. A. Kotowicz, M. A. Sajjad, and J. A. Pasco, "Bone turnover markers in men and women with impaired fasting glucose and diabetes," Calcified Tissue International, vol. 104, no. 6, pp. 599-604, 2019.

[21] E. Lerchbaum, V. Schwetz, M. Nauck, H. Völzke, H. Wallaschofski, and A. Hannemann, "Lower bone turnover markers in metabolic syndrome and diabetes: the population-based study of health in Pomerania," Nutrition, Metabolism, and Cardiovascular Diseases, vol. 25, no. 5, pp. 458-463, 2015.

[22] H. Shu, Y. Pei, K. Chen, and J. Lu, "Significant inverse association between serum osteocalcin and incident type 2 diabetes in a middle-aged cohort," Diabetes/Metabolism Research and Reviews, vol. 32, no. 8, pp. 867-874, 2016.

[23] A. Tan, Y. Gao, X. Yang et al., "Low serum osteocalcin level is a potential marker for metabolic syndrome: results from a Chinese male population survey," Metabolism, vol. 60, no. 8, pp. 1186-1192, 2011.

[24] X. Y. Ma, F. Q. Chen, H. Hong, X. J. Lv, M. Dong, and Q. Y. Wang, "The relationship between serum osteocalcin concentration and glucose and lipid metabolism in patients with type 2 diabetes mellitus - the role of osteocalcin in energy metabolism," Annals of Nutrition and Metabolism, vol. 66, no. 2-3, pp. 110-116, 2015.

[25] Y. Chen, Q. Zhao, G. Du, and Y. Xu, "Association between serum osteocalcin and glucose/lipid metabolism in Chinese Han and Uygur populations with type 2 diabetes mellitus in Xinjiang: two cross-sectional studies," Lipids in Health and Disease, vol. 16, no. 1, p. 139, 2017.

[26] N. C. Thalassinos, P. Hadjiyanni, M. Tzanela, C. Alevizaki, and D. Philokiprou, "Calcium metabolism in diabetes mellitus: effect of improved blood glucose control," Diabetic Medicine, vol. 10, no. 4, pp. 341-344, 1993.

[27] P. Vestergaard, L. Rejnmark, and L. Mosekilde, "Relative fracture risk in patients with diabetes mellitus, and the impact of insulin and oral antidiabetic medication on relative fracture risk," Diabetologia, vol. 48, no. 7, pp. 1292-1299, 2005.

[28] R. Reyes-García, P. Rozas-Moreno, G. López-Gallardo et al., "Serum levels of bone resorption markers are decreased in patients with type 2 diabetes," Acta Diabetologica, vol. 50, no. 1, pp. 47-52, 2013.

[29] M. Yamamoto, T. Yamaguchi, K. Nawata, M. Yamauchi, and T. Sugimoto, "Decreased PTH levels accompanied by low bone formation are associated with vertebral fractures in postmenopausal women with type 2 diabetes," Journal of Clinical Endocrinology and Metabolism, vol. 97, no. 4, pp. 1277-1284, 2012. 
[30] L. Gennari, D. Merlotti, R. Valenti et al., "Circulating sclerostin levels and bone turnover in type 1 and type 2 diabetes," Journal of Clinical Endocrinology and Metabolism, vol. 97, no. 5, pp. 1737-1744, 2012.

[31] S. K. Wong, K. Y. Chin, F. H. Suhaimi, F. Ahmad, and S. ImaNirwana, "Effects of metabolic syndrome on bone mineral density, histomorphometry and remodelling markers in male rats," PloS One, vol. 13, no. 2, article e0192416, 2018.

[32] H. Vlassara, "Recent progress in advanced glycation end products and diabetic complications," Diabetes, vol. 46, Supplement_2, pp. S19-S25, 1997.

[33] K. T. Tonks, C. P. White, J. R. Center, D. Samocha-Bonet, and J. R. Greenfield, "Bone turnover is suppressed in insulin resistance, independent of adiposity," Journal of Clinical Endocrinology and Metabolism, vol. 102, no. 4, pp. 11121121, 2017.

[34] G. E. Hein, "Glycation endproducts in osteoporosis-is there a pathophysiologic importance?," Clinica Chimica Acta, vol. 371, no. 1-2, pp. 32-36, 2006.

[35] S. Kume, S. Kato, S. Yamagishi et al., "Advanced glycation endproducts attenuate human mesenchymal stem cells and prevent cognate differentiation into adipose tissue, cartilage, and bone," Journal of Bone and Mineral Research, vol. 20, no. 9, pp. 1647-1658, 2005.

[36] N. Ogawa, T. Yamaguchi, S. Yano, M. Yamauchi, M. Yamamoto, and T. Sugimoto, "The combination of high glucose and advanced glycation end-products (AGEs) inhibits the mineralization of osteoblastic MC3T3-E1 cells through glucose-induced increase in the receptor for AGEs," Hormone and Metabolic Research, vol. 39, no. 12, pp. 871-875, 2007.

[37] T. Yamamoto, K. Ozono, A. Miyauchi et al., "Role of advanced glycation end products in adynamic bone disease in patients with diabetic nephropathy," American Journal of Kidney Diseases, vol. 38, no. 4, pp. S161-S164, 2001.

[38] N. Napoli, On behalf of the IOF Bone and Diabetes Working Group, M. Chandran et al., "Mechanisms of diabetes mellitus-induced bone fragility," Nature Reviews Endocrinology, vol. 13, no. 4, pp. 208-219, 2017.

[39] W. D. Leslie, M. R. Rubin, A. V. Schwartz, and J. A. Kanis, "Type 2 diabetes and bone," Journal of Bone and Mineral Research, vol. 27, no. 11, pp. 2231-2237, 2012.

[40] N. G. Kondegowda, R. Fenutria, I. R. Pollack et al., "Osteoprotegerin and denosumab stimulate human beta cell proliferation through inhibition of the receptor activator of NF- $\kappa \mathrm{B}$ ligand pathway," Cell Metabolism, vol. 22, no. 1, pp. 77-85, 2015.

[41] M. Ferron, J. Wei, T. Yoshizawa et al., "Insulin signaling in osteoblasts integrates bone remodeling and energy metabolism," Cell, vol. 142, no. 2, pp. 296-308, 2010.

[42] I. G. Poornima, R. H. Mackey, A. M. Buhari, J. A. Cauley, K. A. Matthews, and L. H. Kuller, "Relationship between circulating serum osteoprotegerin and total receptor activator of nuclear kappa-B ligand levels, triglycerides, and coronary calcification in postmenopausal women," Menopause, vol. 21, no. 7, pp. 702-710, 2014.

[43] P. Ducy, M. Amling, S. Takeda et al., "Leptin inhibits bone formation through a hypothalamic relay: a central control of bone mass," Cell, vol. 100, no. 2, pp. 197-207, 2000.

[44] N. Napoli, C. Pedone, P. Pozzilli, F. Lauretani, L. Ferrucci, and R. A. Incalzi, "Adiponectin and bone mass density: the InCHIANTI study," Bone, vol. 47, no. 6, pp. 1001-1005, 2010.
[45] M. Sorocéanu, D. Miao, X. Bai, H. Su, D. Goltzman, and A. Karaplis, "Rosiglitazone impacts negatively on bone by promoting osteoblast/osteocyte apoptosis," Journal of Endocrinology, vol. 183, no. 1, pp. 203-216, 2004.

[46] Y. K. Loke, S. Singh, and C. D. Furberg, "Long-term use of thiazolidinediones and fractures in type 2 diabetes: a metaanalysis," CMAJ: Canadian Medical Association Journal, vol. 180, no. 1, pp. 32-39, 2009.

[47] N. B. Watts, J. P. Bilezikian, K. Usiskin et al., "Effects of canagliflozin on fracture risk in patients with type 2 diabetes mellitus," Journal of Clinical Endocrinology and Metabolism, vol. 101, no. 1, pp. 157-166, 2016.

[48] M. Monami, B. Cresci, A. Colombini et al., "Bone fractures and hypoglycemic treatment in type 2 diabetic patients: a casecontrol study," Diabetes Care, vol. 31, no. 2, pp. 199-203, 2008.

[49] A. V. Schwartz, E. Vittinghoff, D. E. Sellmeyer et al., "Diabetesrelated complications, glycemic control, and falls in older adults," Diabetes Care, vol. 31, no. 3, pp. 391-396, 2008.

[50] G. Mabilleau, M. Pereira, and C. Chenu, "Novel skeletal effects of glucagon-like peptide-1 (GLP-1) receptor agonists," Journal of Endocrinology, vol. 236, no. 1, pp. R29-R42, 2018.

[51] K. Naylor and R. Eastell, "Bone turnover markers: use in osteoporosis," Nature Reviews Rheumatology, vol. 8, no. 7, pp. 379-389, 2012.

[52] M. J. Seibel, "Biochemical markers of bone turnover: part I: biochemistry and variability," Clinical Biochemistry, vol. 26, no. 4, pp. 97-122, 2005.

[53] M. Ogata, N. Iwasaki, R. Ide et al., "Role of vitamin D in energy and bone metabolism in postmenopausal women with type 2 diabetes mellitus: a 6-month follow-up evaluation," Journal of Diabetes Investigation, vol. 9, no. 1, pp. 211-222, 2018.

[54] P. Szulc, F. Munoz, F. Marchand, M. C. Chapuy, and P. D. Delmas, "Role of vitamin D and parathyroid hormone in the regulation of bone turnover and bone mass in men: the MINOS study," Calcified Tissue International, vol. 73, no. 6, pp. 520530, 2003. 